\title{
Sophisticated Resource Orchestration and Incorporating in ATOM
}

\author{
Sumagna Patnaiak, M. Uday kiran, Ch. Mounika, V. Harika
}

\begin{abstract}
The rise of Infrastructure as a Service system brings new chances that conjointly go with newdifficulties in auto-scaling, asset allotment, and security. A basic test supporting these issues is that the consistent following and observing of asset utilization inside the framework. This paper, we will in general present $A T O M, A$ productive and compelling structure to naturally track, screen, and arrange asset utilization in an Infrastructure as a Service (IaaS) framework that is wide utilized in cloud foundation. we will in general utilize novel trailing strategy to constantly follow fundamental framework use measurements with low overhead and build up a Principal part Analysis (PCA) essentially based way to deal with perpetually screen and naturally see inconsistencies bolstered the approximated trailing results. we will in general demonstrate an approach to powerfully set the trailing limit upheld the discovery results, and that's only the tip of the iceberg, an approach to manage trailing standard to affirm its optimality underneath unique outstanding tasks at hand. In conclusion, when potential oddities square measure known, we will in general use thoughtfulness instruments to perform memory legal sciences on VMs guided by dissected outcomes from trailing and checking to spot vindictive conduct inside a VM. we will in general exhibit the extensibility of ATOM through virtual machine (VM) bundle. The execution of our structure is assessed in AN open supply IaaS framework.
\end{abstract}

Keywords : Infrastructure as a Service, cloud, following, checking, peculiarity recognition, virtual machine thoughtfulness.

\section{INTRODUCTION}

Molecule is a free and open-source [5] content and source code supervisor for macOS, Linux, and Microsoft Windows [6] with help for modules written in Node.js, and installed Git Control, created by GitHub. Molecule is an area for working application constructed utilizing web technologies.[7] Most of the broadening bundles have free programming licenses and are network assembled and maintained.[8] Atom depends on Electron,[9] a structure that empowers cross-stage work area applications utilizing Chromium and Node.js.[10] It is written in CoffeeScript and Less.[12] It can be similarly utilized as a coordinated improvement condition (IDE).[13] Atom was discharged from beta, as adaptation 1.0, on 25 June 2015.[17] Its designers consider it a "hackable content tool for the 21st Century".Security is another principal framework. For instance, it was accounted for saries assaulted Amazon cloud by administration (DDoS) bots on client VMs by in Elasticsearch [2]. Asset utilization bits of knowledge to

Revised Version Manuscript Received on 16 September, 2019.

* Correspondence Author

Dr.SumagnaPatnaiak - Professor JBIET

M. UdayKiran-Assistant Professor JBIET

Ch.Mounika - Assistant Professor JBIET

V.Harika - Assistant Professor JBIET address security concerns. to continually screen asset utilization for asset designation, as well as in the framework. As of not long ago, the prescribed procedures for alleviating DDoS and different assaults in AWS incorporate utilizing CloudWatch to make straightforward edge cautions on observed measurements and ready clients for potential assaults [3]. In our work we demonstrate to identify the abnormalities consequently while sparing clients the inconvenience on setting enchantment edge esteems.

A persuading precedent Eucalyptus is a paid and open-source PC programming for building Amazon Web Services (AWS)perfect private and mixture distributed computing situations, initially created by the organization Eucalyptus Systems. Eucalyptus is an abbreviation for Elastic Utility Computing These perceptions show that a major test supporting a few vital issues in an IaaS framework is the ceaseless following and observing of asset use in the framework. Besides, a few applications additionally require wise and computerized coordination of framework assets, by going past aloof following and checking, and presenting auto-recognition of anomalous conduct in the framework, and dynamic contemplation and adjustment once abnormality has been recognized and affirmed. This persuades us to plan and execute ATOM, a proficient and compelling structure to consequently follow, arrange, and screen asset use in an IaaS framework. Engineering for Linking Your Programs To Useful Systems.[2] Eucalyptus empowers pooling figure, stockpiling, and system assets that can be progressively scaled up or down as application remaining tasks at hand change.[3] Mårten Mickos was the CEO of Eucalyptus.[4] In September 2014, Eucalyptus was obtained by Hewlett-Packard and afterward kept up by DXC Technology. Eucalyptus gives an AWS-like administration called CloudWatch.CloudWatch can screen asset use of each VM. To lessen overhead, such information is just gathered from each VM at consistently, and after that answered to the CLC through a CC. Clearly,gathering asset use progressively presents overhead in the frameworks. At the point when there are a lot of VMs to screen, the issue turns out to be much more appalling and will convey huge overhead to the system. CloudWatch tends to this issue by gathering estimations just once consistently, yet this gives just a discrete, inspected perspective of the framework status and isn't adequate to giving ceaseless comprehension and insurance of the framework. Another constraint in existing methodologies like CloudWatch is that they just do uninvolved checking. No dynamic online asset organization is set up towards distinguishing framework inconsistencies, potential dangers and assaults. We see that, e.g., in the previously mentioned DDoS assault to Amazon cloud, disturbing signs can be gained naturally from asset utilization 
information, which are promptly to dissect with no pre-handling like framework logs [6]. Dynamic online asset observing and coordination is extremely valuable in accomplishing an increasingly secure and dependable framework. Dynamic online asset checking gives us the chances to trigger VM reflection to investigate the framework and make sense of what has conceivably turned out badly. The reflection into VMs then permits to organize asset utilization and designation in the IaaS framework to accomplish a progressively secure framework as well as better execution. Note that VM reflection is costly. Without content following and internet checking and organization, it is relatively difficult to make sense of when to do VM contemplation and what explicit focus to introspect in a host VM. We will probably computerize this procedure and trigger VM reflection just when required. We allude to this procedure as asset organization. Particle presents a web based following module that keeps running at $\mathrm{NC}$ and persistently tracks different execution measurements and asset use estimations of all VMs. The CLC is indicated as the tracker, and the NCs are signified as the eyewitnesses. The objective is to supplant the inspected view at the CLC with a nonstop comprehension of framework status, with least overhead.Particle at that point utilizes a computerized checking module that consistently screens the asset use information announced by the web based following module. The objective is to recognize peculiar things by mining the asset use information. This is particularly useful for distinguishing assaults that could cause changes in asset use, for instance, one VM devours every accessible asset and starves all different VMs running on the equivalent physical PC [7]. The gauge for internet checking is to just characterize an edge an incentive for any measurement of intrigue. Plainly, this methodology isn't extremely successful against dynamic and complex assaults and irregularities. Particle utilizes a dynamic web based observing technique that is created dependent on PCA. We plan a PCA-based technique that consistently breaks down the predominant subspace characterized by the estimations from the following module, and consequently raises an alert at whatever point a move in the overwhelming subspace has been recognized. Even though PCA-based techniques have been utilized for peculiar recognition in different settings, another test in our setting is to adapt to surmised estimations created by web based following, and structure strategies that can naturally adjusting to and modifying the following mistakes.In conclusion, virtual machine contemplation (VMI) is utilized to recognize and distinguish malevolent conduct inside a VM. VMI procedures, for example, investigating VM memory space will in general be of extraordinary expense. On the furthur chance that we don't know where and when an assault may have happened,we should experience the whole memory always, which is obviously costly, particularly if VMs to be examined are such many. The principal alternative is to set a limit for every asset utilization measure, and we consider there might be an inconsistency if the announced esteem is past the edge for that measure and trigger a VMI. This is the technique that current frameworks like AWS and Eucalyptus have received for auto scaling undertakings. The second alternative is to utilize the web based observing technique in the checking module to consequently identify abnormality and trigger a VMI, and in addition managing the contemplation to explicit areas in the VM memory space dependent on the information from web based observing and following. We indicate the second technique as coordination.All things considered, take note of that ATOM is a conclusion to-end system that coordinates internet following, web based observing, and arrangement into one structure, while UBL centers around irregularity discovery in execution information without the mix of following and organization. Consequently, UBL is "proportionate " to the observing segment in ATOM.

\section{RELATED WORK}

To the best of our insight, none of existing IaaS stages can give constant following, observing, and organization of framework asset use. Besides, none of them can do astute, mechanized checking for a substantial number of VMs and do coordination inside a VM. Inside these systems, there are basic requirements for the controller to consistently gather asset use information and screen framework wellbeing. AWS [1] and Eucalyptus [4], [5] use CloudWatch administration to screen VMs and different parts in some settled interims, e.g., consistently. This gives cloud clients a framework wide perceivability into asset usage and enables clients to set some straightforward limit-based cautions to screen and guarantee framework wellbeing. OpenStack is building up a venture called Ceilometer, to gather assets use estimations. Be that as it may, these methodologies just give a discrete, examined perspective of the framework. A few developing new businesses, for example, DATADOG and libra to could screen in an all the more fine-grained granularity, gave the required virtual products are introduced. Be that as it may, this acquaints more system overhead with the cloud, which turns out to be more terrible when the checked foundation scales up. Despite what might be expected, ATOM altogether lessens the system overhead by using the ideal web based following calculation, while giving pretty much a similar measure of data. Moreover, all these cloud observing administrations offer exceptionally constrained capacity in checking and guaranteeing framework wellbeing. UBL [8] utilizes gathered VM use information to prepare Self-Organizing Maps for peculiarity forecast, which fills a comparative need to ATOM's observing part. Other than the point by point examination in Section 1, SOM requires an express preparing stage and should be prepared by typical information, while PCA could distinguish what is ordinary specifically from the history information gave ordinary information is the dominant part. Not at all like UBL and ATOM which just require VM utilization information, PerfCompass gathers framework consider follows and checks the execution units being influenced to distinguish whether a VM execution irregularity is caused by interior blame like programming bugs, or from an outer source, for example, coinciding VMs. Astrolabe is an observing administration for disseminated re-sources, to perform client characterized total (e.g. number of hubs that fulfill certain property) on-the-fly for the host hello there erarchy. It is proposed as an "outlining system". Like Astrolabe, SDIMS is another framework that totals data about extensive scale arranged frameworks with better capacity, adaptability, and authoritative disconnection. Ganglia is a universally useful versatile dispersed observing framework for elite processing frameworks which additionally has a various leveled configuration to screen and total the hubs and has been utilized in 
numerous bunches. These endeavors are like the CloudWatch module right now utilized in AWS/Eucalyptus, and they diminish observing overhead by straightforward collections. While the motivation behind ATOM's following module is to lessen information exchange, however it does as such utilizing web based following rather than basically accumulating which conveys considerably more fine-grained data. STAR is a various leveled calculation for adaptable conglomeration that decreases correspondence overhead via cautiously circulating the permitted blunder spending plans. It suites frameworks like SDIMS well. InfoEye is a model-based data the executives framework for vast scale benefit overlay organizes through a lot of observing sensors conveyed on various overlay hubs with lessened overhead accomplished by specially appointed conditions channels. InfoTrackis a checking framework that is like ATOM's following module, in that it endeavors to limit constant observing expense with most data accuracy saved, by utilizing fleeting and spatial connection of observed qualities, while ATOM utilizes an ideal web based following calculation that is demonstrated to accomplish the best sparing in system cost with no earlier learning on the information. MELA is a checking structure for cloud benefit which gathers diverse elements of information custom fitted for investigating cloud flexibility reason. Molecule may utilize MELA to gather, track, and screen diverse kinds of measurements than those officially accessible through CloudWatch. Cloud security. IaaS framework additionally presents to us another arrangement of security issues. Driving cloud suppliers have created propelled system toGuarantee the security of their IaaS frameworks. AWS has many worked in security highlights, for example, firewalls, encoded capacity and security logs. OpenStack utilizes a security segment called Keystone to do confirmation and approval. It likewise has security rules for system correspondence in its system part Neutron. Different IaaS stages have comparable security arrangements, which are chiefly firewalls and security gatherings. All things considered, it is yet conceivable that programmers could sidestep known security arrangements, or cloud clients may incidentally run a few malicious programming. It is in this way basic to have the capacity to distinguish such peculiarity in close ongoing to abstain from leaving programmers a lot of time to cause critical harm. Thus we require a checking arrangement that could effectively distinguish abnormality, and recognize possibly vindictive conduct over countless examples. AWS as of late receives its CloudWatch benefit for DDoS assaults [3], however it re-quires client to check recorded information and set an "enchantment esteem" as the limit physically, which is doubtful if client's fundamental remaining tasks at hand change habitually. Conversely, ATOM could consequently take in the typical conduct from past observed information, and distinguish increasingly complex assaults other than DDoS assaults utilizing PCA. PCA has been generally used to identify abnormality in system traffic volume in spine systems [12]. A virtual machine assault is viewed as a noteworthy security danger. Particle's reflection segment use existing open source VMI apparatuses, for example, Stackdb [10] and Volatility [18] to pinpoint the oddity to the correct procedure. VMI is an outstanding strategy for guaranteeing VM security. It has additionally been concentrated for IaaS frameworks. Be that as it may, to always anchor VM utilizing VMI technique, the whole VM memory should be crossed and broke down occasionally. It might likewise require the VM to be suspended so as to access VM memory. Blacksheep [19] is such a framework, to the point that recognizes rootkit by dumping and looking at gatherings of comparative machines. Even though the execution overhead is professed to be acceptably low to help constant observing, plainly client projects will be contrarily influenced. Another arrangement was recommended for cloud clients to confirm the trustworthiness of their VMs. In any case, this isn't a "functioning discovery and response" framework. Conversely, ATOM empowers activating VMI just when a potential assault is recognized, and it likewise finds the applicable memory area to investigate and introspect significantly more adequately and proficiently utilizing its organization segment.

\section{PROPOSE SYSTEM}

(1) Tracking segment: ATOM adjusts the ideal web based following calculation for one-measurement web-based following inside the checking administration on NCs. This drastically lessens the over-make a beeline for screen cloud assets and empowers nonstop estimations to CC and CLC;

(2) Monitoring segment (peculiarity location): ATOM adds this part in CLC to break down following outcomes by the following segment, which gives persistent asset utilization information progressively. It utilizes an adjusted PCA technique to consistently follow the partitioned subspace, as characterized by the multi-dimensional qualities from the following outcomes, and consequently finding peculiarity by identifying outstanding movement in the fascinating subspace. It likewise creates abnormality data for further examination by the coordination component when this occurs. The checking segment additionally alters the following edge from the respective part progressively online dependent on the information patterns and an ideal false caution rate.

(3) Orchestration part (thoughtfulness and investigating): when a potential abnormality is recognized by the checking component, an Introspect ask for alongside irregularity data is sent to the organization segment on $\mathrm{NC}$, in which VMI instruments, and VM troubleshooting devices are utilized to distinguish the bizarre conduct inside a VM and raise an alert to cloud clients for further examination.

\section{ATOM Frame Work}

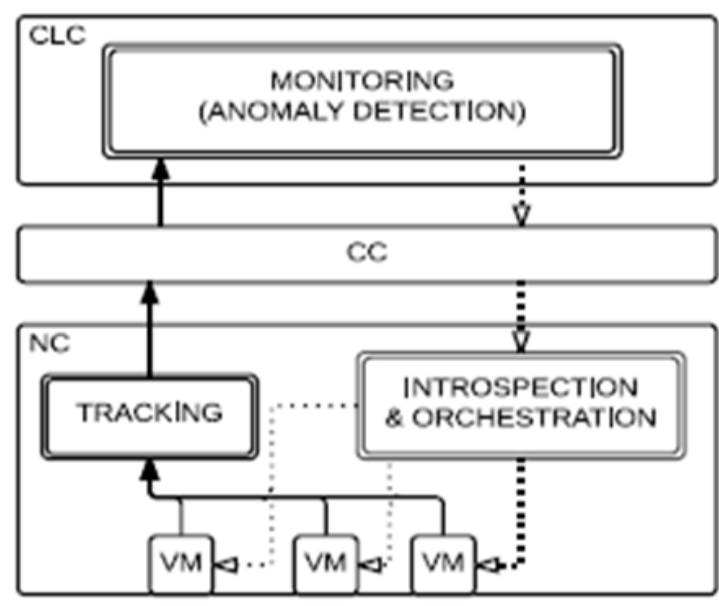

Fig 1: ATOM System 


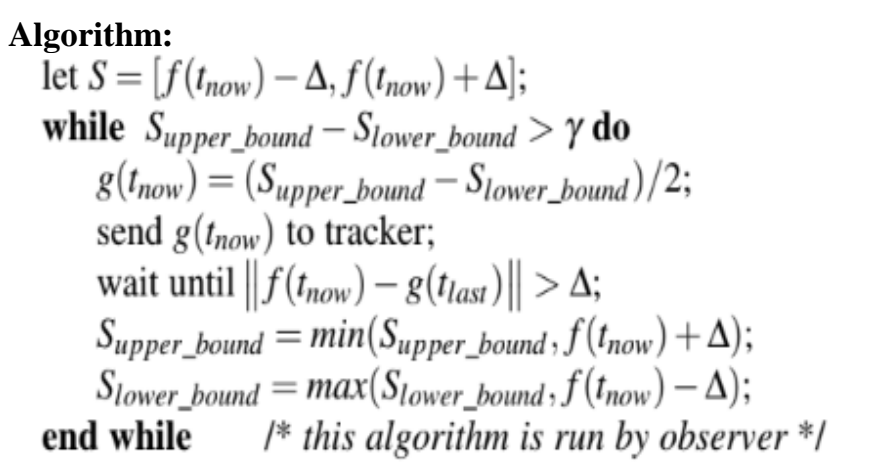

\section{One round of online tracking for real values}

\section{CONCLUSIONS}

We show the ATOM-system that can be adequately consolidated into a standard IaaS structure to give motorized, steady following, checking, and coordination of structure resource use in about continuous. Iota is to an incredible degree significant for variation from the norm recognizable proof, auto-scaling, and dynamic resource assignment and load altering in IaaS systems. Fascinating future work consolidates growing ATOM for further developed expedient arrangement and joining the obstruction against impressively increasingly many-sided attacks in ATOM.

\section{REFERENCES}

[1]. Eucalyptus. http://www8.hp.com/us/en/cloud/helioneucalyptus. html. Accessed Nov. 5, 2016.

[2]. D Nurmi, R. Wolski, C. Grzegorczyk, G. Obertelli, S. Soman, L. Yous-eff, and D. Zagorodnov, "The eucalyptus open-source cloud-computing system," in CCGRID, 2009.

[3]. M Du and F. Li, "Spell: Streaming parsing of system event logs," in ICDM, 2016.

[4]. W Dawoud, I. Takouna, and C. Meinel, "Infrastructure as a service security: Challenges and solutions," in INFOS, 2010.

[5]. H. Ringberg, A. Soule, J. Rexford, and C. Diot, "Sensitivity of PCA for traffic anomaly detection," in SIGMETRICS Performance Evaluation Review, 2007.

[6]. A. Lakhina, M. Crovella, and C. Diot, "Diagnosing network-wide traffic anomalies," in SIGCOMM, 2004.

[7]. V. Varadarajan, T. Kooburat, B. Farley, T. Ristenpart, and M. M. Swift, "Resource-freeing attacks: improve your cloud performance (at your neighbor's expense)," in CCS, 2012.

[8]. W. Li, H. H. Yue, S. Valle-Cervantes, and S. J. Qin, "Recursive PCA for adaptive process monitoring," Journal of process control, 2000.

[9]. D J. Dean, H. Nguyen, and X. Gu, "UBL: Unsupervised behavior learn-ing for predicting performance anomalies in virtualized cloud systems," in ICAC, 2012.

[10]. LibVMI. http://libvmi.com/. Accessed Nov. 5, 2016.

[11]. D. Johnson, M. Hibler, and E. Eide, "Composable multi-level debugging with Stackdb," in VEE, 2014.

[12]. K. Yi and Q. Zhang, "Multi-dimensional online tracking," in SODA, 2009.

[13]. Amazon. http://www.aws.amazon. com/.Accessed Nov. 5, 2016.

[14].ITWORLD.http://www.itworld.com/se-curity/428920/attackers-install -ddos-bots-amazon-cloud exploitingelastic search weakness. AccessedNov. 5, 2016

[15]. Amazon. AWS Best Practices for DDoSResiliency. https://d0.awsstatic.com/whitepapers/DDoS White PaperJune2015.pdf. Accessed Nov. 5, 2016.

[16]. J. E. Jackson and G. S. Mudholkar, "Control procedures for residuals associated with principal component analysis," Technometrics, 1979.

[17]. L. Huang, M. I. Jordan, A. Joseph, M. Garofalakis, and N. Taft, "In-network PCA and anomaly detection," in NIPS, 2006.

[18]. Volatility. http://www.volatilityfoundation.org/. Accessed Nov. 5, 2016.

[19]. A. Bianchi, Y. Shoshitaishvili, C. Kruegel, and G. Vigna, "Blacksheep: detecting compromised hosts in homogeneous crowds," in CCS, 2012
[20]. M. Ester, H.-P. Kriegel, J. Sander, X. Xu et al., “A density-based algorithm for discovering clusters in large spatial databases with noise." in KDD, 1996.

[21]. D. E. Difallah, A. Pavlo, C. Curino, and P. Cudre Mauroux, "OLTP-Bench: An extensible testbed for benchmarking relational databases," PVLDB, 2013.

[22].

StackDB http://www.flux.utah.edu/software/stackdb/doc/all.html\#using-eucaly ptus-to-run-qemukvm. Accessed Nov. 5, 2016.

[23]. I. Goiri, R. Bianchini, S. Nagarakatte, and T. D. Nguyen, "Approx-hadoop: Bringing approximations to mapreduce frameworks," in ASP-LOS, 2015. 